\title{
Strain response of POF sensors
}

\author{
Ada Abang*a, David J. Webb ${ }^{\mathrm{a}}$, and Peng Gang-Ding ${ }^{\mathrm{b}}$ \\ ${ }^{a}$ Aston Institute of Photonic Technologies, Aston University, Birmingham, B4 7ET, UK \\ ${ }^{\mathrm{b}}$ School of Electrical Engineering and Telecommunications, University of New South Wales \\ Sydney, NSW 2052, Australia
}

\begin{abstract}
With regard to polymer fibre Bragg gratings, we investigate one of the consequences of the visco-elastic nature of the constituent polymer: hysteresis in the response of wavelength shift vs sensor elongation. We show that when a grating sensor is directly bonded to a substrate, the hysteresis is reduced by a factor of 10 from the case where the sensor is freely suspended between two supports.
\end{abstract}

Keywords: Polymer optical fibre, PMMA, fibre Bragg grating, strain sensor

\section{Introduction}

Fibre Bragg grating sensors in polymer fibre are attracting interest because of their different material properties compared to silica ${ }^{1}$. For example, they can survive higher strains and they possess a much lower Young's modulus and therefore perturb any structure to which they are attached rather less than does the comparatively stiff silica fibre; this is important when the structure is itself rather compliant, for example as is the case with a tapestry ${ }^{2}$. A potentially serious problem with polymer optical fibre Bragg grating (POFBG) sensors relates to the viscoelastic nature of the material. When a sizeable elongation is applied to the sensor and then released, there can remain a significant wavelength shift which only gradually relaxes over time. For example, in one experiment, a strain of $5 \%$ was applied by tensioning a POFBG sensor between two supports, one of which was mounted on a translation stage, resulting in a wavelength shift of $72 \mathrm{~nm}$. After 30 minutes the tension was removed but a wavelength shift of about $20 \mathrm{~nm}$ remained $^{3}$.

It should be recognised however that when grating sensors are actually applied to the monitoring of strain in a structure, they are not normally fixed at points either side of the sensor; instead the sensor would usually be glued directly to the structure, or possibly embedded in it. In this work we investigate whether this method of fixation can mitigate the effects of the viscoelastic nature of the POF.

\section{Experiments}

Two identical fibre Bragg gratings were fabricated in a PMMA based single mode POF which had an outer diameter of about $180 \mu \mathrm{m}$ and a core diameter of $10 \mu \mathrm{m}$. A helium-cadmium laser with a wavelength of $325 \mathrm{~nm}$ and an ultraviolet (UV) power output of $30 \mathrm{~mW}$ was used to inscribe the Bragg gratings in the POF. The laser beam was focused vertically downwards using a $10 \mathrm{~cm}$ focal length cylindrical lens, through a $1034.2 \mathrm{~nm}$ period phase mask and on to the fibre ${ }^{4}$. The single-mode POF was laid on a v-groove and taped down using polyimide tape. This type of fibre has an average inscription time of 7 minutes. The Bragg wavelength of the inscribed grating was in the region of $1530 \mathrm{~nm}$ with a grating length of $2 \mathrm{~mm}$, determined by the width of the UV laser beam.

Inscription was monitored using a 1550nm single mode 50:50 silica coupler, a broadband light source (Thorlabs ASEFL7002-C4) and an optical spectrum analyzer (OSA). A connection was made between the arm of the silica coupler and the POF using a FC/APC connector on the silica fibre which was then butt coupled to the POF. A small amount of polymer index matching gel was used in the coupling to reduce Fresnel reflections.

abangam@aston.ac.uk

OFS2012 22nd International Conference on Optical Fiber Sensors, edited by

Yanbiao Liao, Wei Jin, David D. Sampson, Ryozo Yamauchi, Youngjoo Chung, Kentaro Nakamura, Yunjiang Rao,

Proc. of SPIE Vol. 8421, 84214O - (C) 2012 SPIE · CCC code: 0277-786/12/\$18 - doi: 10.1117/12.974946

Proc. of SPIE Vol. $8421842140-1$ 
High-quality end faces of the POF are essential for effective butt-coupling; these were prepared using an $80^{\circ} \mathrm{C}$ hot cleave as described by Abdi et $a l^{5}$. Following inscription, the POFBG was spliced to a step index silica fibre using Norland 78 UV curing optical adhesive and Omni Cure Series 1000, UV curing light source as described by Johnson et $\mathrm{al}^{3}$.

\subsection{Strain sensor suspended in free space}

One end of one of the FBG sensors was glued to a stationary stage and the other end to a translation stage using the UV curing glue and curing light source as shown in figure 1. The distance between the two stages where the POF was suspended with no slack was $45 \mathrm{~mm}$. The POF was strained to $2.3 \%$ of this distance, which is $1 \mathrm{~mm}$. The strain was increased manually in steps of $0.1 \mathrm{~mm}$ at an interval of 30 seconds. The POF sensor was left for 1 hour at $2.3 \%$ strain. The sensor was then unstrained at the same interval of 30 seconds. The reflected spectrum of the FBG sensor from the OSA was capture on a PC using the auto-capture mode on the lab-view software as the strain was increased and then decreased.

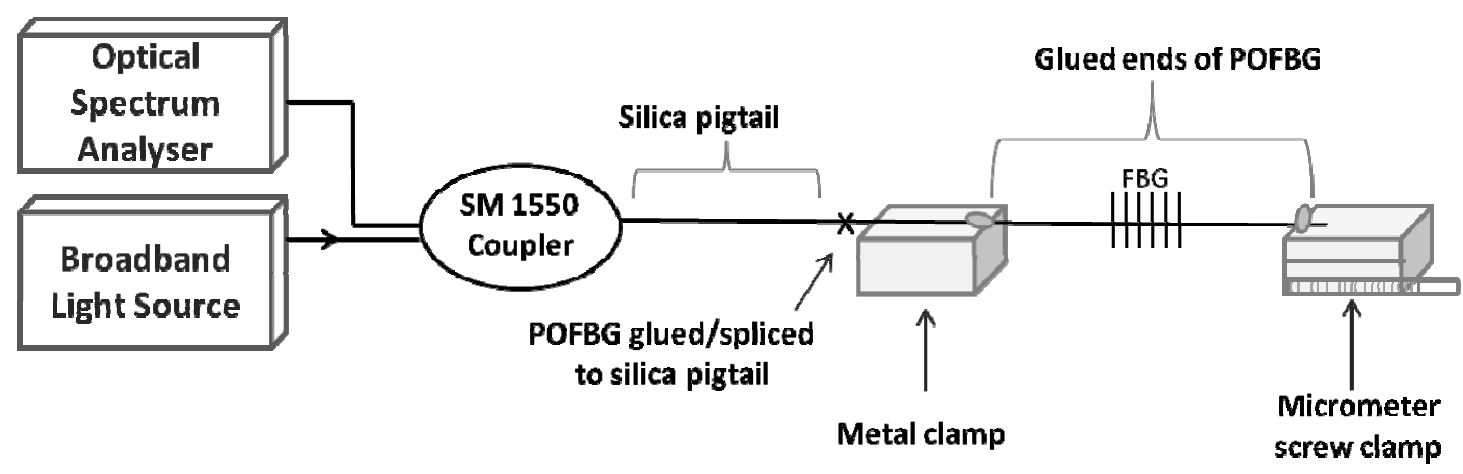

Figure 1. Schematic diagram of POF sensor suspended in free space

The $-3 \mathrm{~dB}$ averaging method ${ }^{6}$ was used to calculate the Bragg wavelength of the spectrum at each strained state. The values obtained from the averaging method were plotted and are shown in figure 2 . At a strain of $2.3 \%$, a wavelength shift of $30.7 \mathrm{~nm}$ was observed, resulting in a strain sensitivity of $1.3 \mathrm{pm} / \mu \varepsilon$. The initial Bragg wavelength of the FBG sensor before the strain was applied was $1536.8 \mathrm{~nm}$ and the wavelength of the sensor when unstrained at the end of the experiment was $1540.8 \mathrm{~nm}$ which results in a wavelength difference of $4.0 \mathrm{~nm}$.

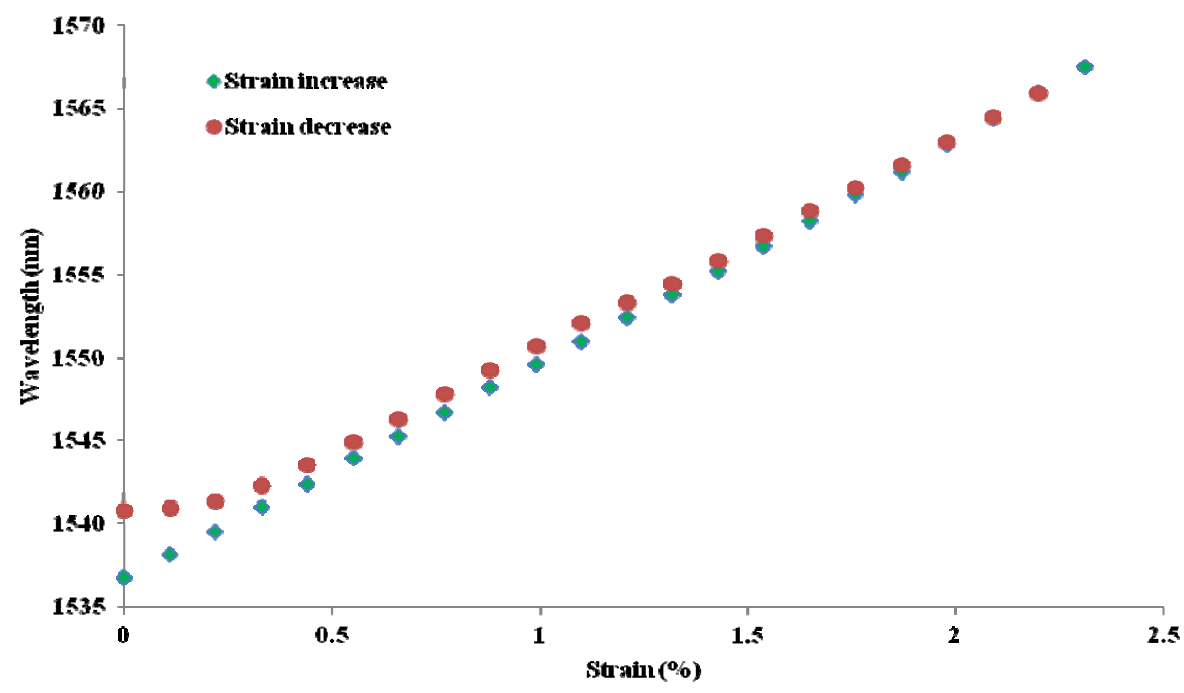

Figure 2. Strain response of POFBG suspended in free space showing hysteresis at lower strain values 


\subsection{Strain sensor UV glued to plastic beam}

The second similar fabicated FBG sensor was UV glued to a plastic (perspex) beam using the UV curing light source. The plastic beam had a total length of $30 \mathrm{~cm}$, it was $3 \mathrm{~cm}$ wide and $0.2 \mathrm{~cm}$ thick. The beam was clamped to an optical bench so that $8 \mathrm{~cm}$ projected beyond the edge of the bench. The point at which the Bragg grating was inscribed on the polymer fibre was placed $2 \mathrm{~cm}$ from the clamped point. Approximately a $2 \mathrm{~mm}$ thickness of UV curing glue was placed from $1 \mathrm{~cm}$ before to $1 \mathrm{~cm}$ after the FBG inscription point along the fibre and cured using the UV curing lamp. A schematic diagram of the setup is shown in figure 3.

A uniformly incremental force was applied at the end of the beam to bend it downwards and hence strain the FBG sensor at 30 seconds time interval. At a strain 2.3\%, the FBG sensor was left for 1 hour. After 1 hour, the force was then decreased uniformly at the same 30 second time intervals. The spectrum of the grating at each time interval was captured using lab view auto capture software. The strain applied on the POF sensor was calculated using equation 1 which was derived from Benham et $\mathrm{al}^{7}$.

$$
\text { Strain }(\varepsilon)=\frac{3 \mathrm{Ddh}}{2 \mathrm{~L}^{3}}
$$

where $\mathrm{D}$ is the distance of the glued POF grating from the free end of the beam, $d$ is the displacement caused by the applied force on the beam, $h$ is the thickness of the beam and $\mathrm{L}$ is the length of the free end of the beam from the clamped point.

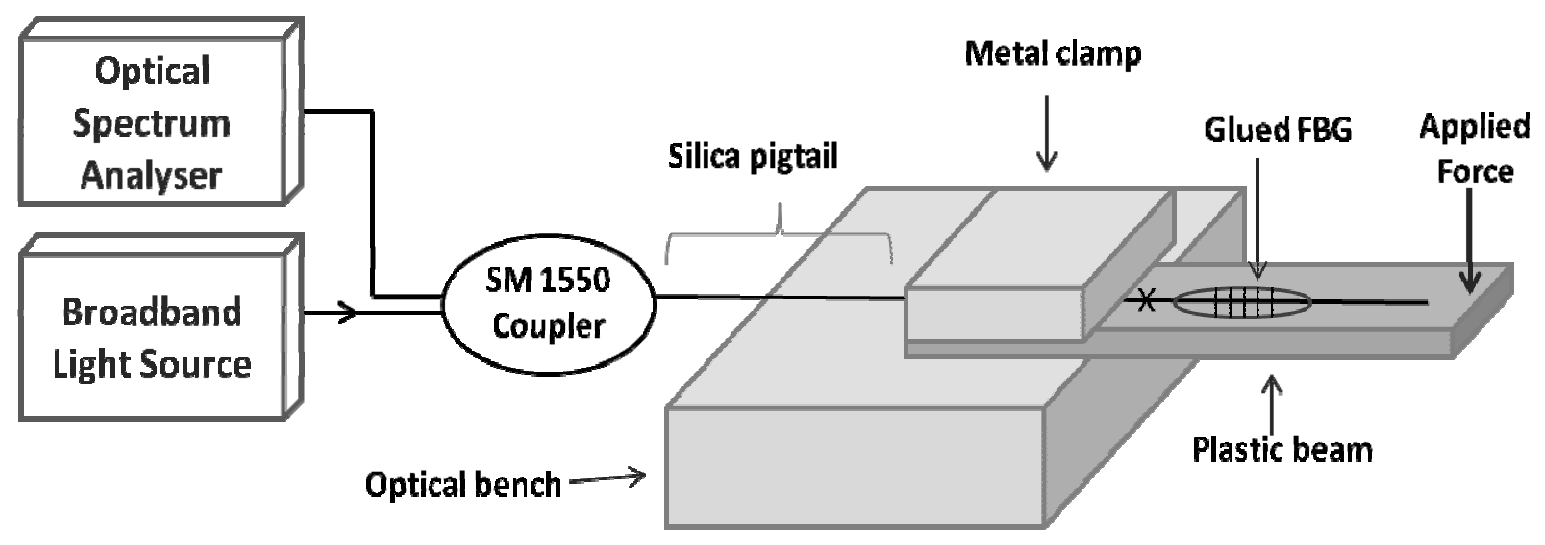

Figure 3. Schematic diagram of POF sensor glued to plastic beam

The $3 \mathrm{~dB}$ averaging method was also used to calculate the Bragg wavelength of the sensor at each point. The plot of the results obtained is shown in figure 4 . A wavelength shift of $29.8 \mathrm{~nm}$ was observed at a strain of $2.3 \%$. The FBG sensor had an initial Bragg wavelength of $1532.1 \mathrm{~nm}$. After the application and removal of strain, the sensor had a wavelength of $1532.7 \mathrm{~nm}$ which shows a difference of $0.6 \mathrm{~nm}$. This difference is more than 6 times smaller than $4.0 \mathrm{~nm}$ which was obtained in the previous experiment where the fibre was suspended in free space. 


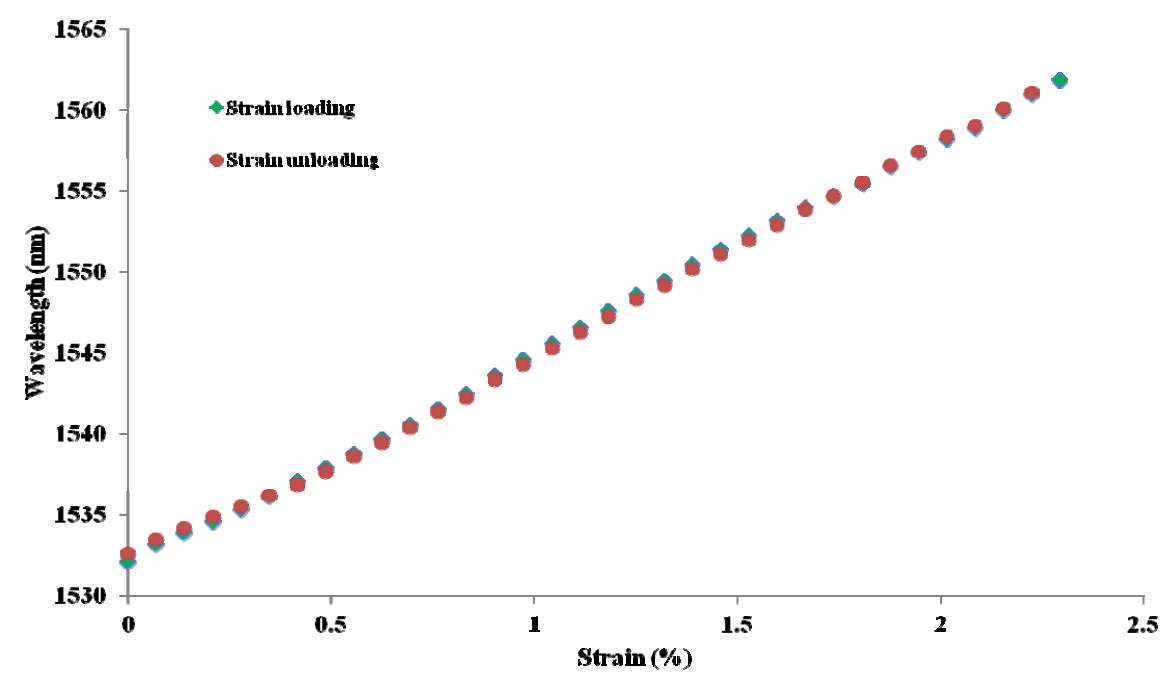

Figure 4. Strain response of POFBG glued to plastic beam with considerably reduced hysteresis

\section{Discussion and Conclusion}

A comparison of figures 3 and 4 shows that the major part of the hysteresis displayed when the fibre is supported at either side of the sensor is removed when the sensor is bonded to a substrate. In this case the beam effectively forces the fibre back to somewhere close to its original length. Examination of figure 4 shows the response is not ideal; there is some non-linearity visible and there is still a small amount of hysteresis. We suspect both of these may be attributed to the plastic beam, which has its own viscoelastic properties.

In conclusion; we have demonstrated for the first time that the hysteresis in the strain response arising from the viscoelastic nature of polymer fibre Bragg grating sensors can be considerably reduced - by a factor of more than 6 when the sensor is directly attached to a structure, as would be the case when such a sensor is used in a real-world application.

\section{References}

[1] Webb D. J. and Kalli, K., "Polymer fibre Bragg gratings," in Fibre Bragg Grating Sensors: Thirty Years from Research to Market, A. Cusano, ed. (Bentham eBooks, 2010).

[2] Ye C. C., Dulieu-Barton J. M., Webb D. J., Zhang C., Peng G. D., Chambers A. R., Lennard F. J., and Eastop D. D., "Applications of polymer optical fibre grating sensors to condition monitoring of textiles," in 20th International Conference on Optical Fibre Sensors, J. D. C. Jones, ed. (SPIE, Edinburgh, 2009), p. 75030M.

[3] Johnson I. P., Webb D. J., Kalli K., Large M. C. and Argyros A., "Multiplexed FBG sensor recorded in multimode microstructured polymer optical fibre." Photonic Crystal Fibres, Brussels - Photonics Europe, SPIE 7714. (2010).

[4] Dobb H., Webb D. J., Kalli K., Argyros A., Large M. C. J., and van Eijkelenborg M. A., "Continuous wave ultraviolet light-induced fibre Bragg gratings in few- and single-mode microstructured polymer optical fibres," Optics Letters 30(24), 3296-3298 (2005).

[5] Abdi, O., K. C. Wong, et al., "Cleaving of solid single mode polymer optical fibre for strain sensor applications." Optics Communications 282(5): 856-861 (2009).

[6] Rivera, E. and D. J. Thomson "Accurate strain measurements with fibre Bragg sensors and wavelength references." Smart Materials and Structures 15: 325-330, (2006).

[7] Benham P. P., Armstrong C. G., Mechanics of Engineering Materials. P. P. Hall. Edinburgh, Pearson Education Limited: 185-191, 235-239 (1996). 